\title{
The organisational and human resource challenges facing primary care trusts: protocol of a multiple case study Elizabeth V Newbronner ${ }^{1}$, Mike J Pedler ${ }^{2}$, J Tim Scott*3 and Trevor A Sheldon4
}

Address: 1Visiting Research Fellow at the Department of Health Studies, University of York UK and Divisional Director, Acton.Shapiro, Consultants in Primary and Community Care, ${ }^{2}$ Revans Professorial Fellow, the Revans Centre for Action Learning, University of Salford, Visiting Professor at the Centre for Leadership Development in the Department of Health Studies, University of York, UK and independent writer, researcher and consultant, 3 Research Fellow at the Department of Health Studies, University of York, UK and 4Head of the Department of Health Studies, University of York, UK

E-mail: Elizabeth V Newbronner - liz@actonshapiro.co.uk; Mike J Pedler - mike@craglea.demon.co.uk; J Tim Scott* - its2@york.ac.uk; Trevor A Sheldon - tas5@york.ac.uk

*Corresponding author

BMC Health Services Research 200I, I:12

Accepted: 28 November 2001

This article is available from: http://www.biomedcentral.com/1472-6963/1/12

(C) 200 I Newbronner et al; licensee BioMed Central Ltd. Verbatim copying and redistribution of this article are permitted in any medium for any noncommercial purpose, provided this notice is preserved along with the article's original URL. For commercial use, contact info@biomedcentral.com

\begin{abstract}
Background: The study is designed to assess the organisational and human resource challenges faced by Primary Care Trusts (PCTs). Its objectives are to: specify the organisational and human resources challenges faced by PCTs in fulfilling the roles envisaged in government and local policy; examine how PCTs are addressing these challenges, in particular, to describe the organisational forms they have adopted, and the OD/HR strategies and initiatives they have planned or in place; assess how effective these structures, strategies and initiatives have been in enabling the PCTs to meet the organisational and human resources challenges they face; identify the factors, both internal to the PCT and in the wider health community, which have contributed to the success or failure of different structures, strategies and initiatives.

Methods: The study will be undertaken in three stages. In Stage I the key literature on public sector and NHS organisational development and human resources management will be reviewed, and discussions will be held with key researchers and policy makers working in this area. Stage 2 will focus on detailed case studies in six PCTs designed to examine the organisational and human resources challenges they face. Data will be collected using semi-structured interviews, group discussion, site visits, observation of key meetings and examination of local documentation. The findings from the case study PCTs will be cross checked with a Reference Group of up to 20 other $\mathrm{PCG} / \mathrm{Ts}$, and key officers working in organisational development or primary care at local, regional and national level. In Stage 3 analysis of findings from the preparatory work, the case studies and the feedback from the Reference Group will be used to identify practical lessons for PCTs, key messages for policy makers, and contributions to further theoretical development.
\end{abstract}

\section{Background}

Primary Care Trusts (PCTs) are new and in many re- spects unique organisations. The self-employed status of the core members of PCTs i.e. general practitioners 
(GPs) sets them apart from most other public and private sector organisations. These new organisations will have to develop more sophisticated organisational structures and methods to facilitate participation and commitment to organisational objectives than would be necessary in other contexts. The recently published final report of a national evaluation of PCG/Ts will been drawn upon to guide the design and conduct of the project.[1]

PCTs face a number of organisational development challenges.[1,2] In particular they will need to:

- Develop an organisational structure that balances day to day management with active and meaningful involvement of health professionals in strategic roles and decision making;

- Engage GPs in the work of the PCT, some of whom may regard PCTs as potential 'mini-health authorities' of little relevance to their day-to-day work, and achieve a balance between the influence of GPs and nurses at executive and board levels;

- Develop stronger relationships with colleagues in partner organisations, in particular social services, the local authority, acute trusts and user groups; [1]

- Develop robust devolved locality structures within PCTs;[1]

- Ensure they can recruit and retain a workforce which has the capacity, skills, diversity and flexibility to meet the demands on the services it provides, including primary care services;

- Develop and implement appropriate HR policies both for staff directly employed by the PCT and those employed at practice level;[3].

- Respond to the context and challenges of Shifting the Balance of and Power and address the major concerns from research about the current lack of organisational and management capacity in PCTs in relation to what is required of them.[1,4,5]

PCG/Ts differ from previous models of primary care commissioning in the NHS, specifically because PCG/T membership is mandatory (previous involvement in commissioning by GPs was voluntary), PCTs are more inclusive of other health and social care professionals, they are larger organisations, and their work must be consistent with the local Health Improvement Programme (HImP) and National Service Frameworks. [6] Whilst individual GPs can choose not to actively participate, the experience of Total Purchasing Pilots (TPPs) suggests that those PCTs with greater levels of involvement are likely to prove more successful in realising their ambitions and achieving service change.[7]

Government policy also emphasises the importance of PCTs and practices working in partnership with staff, patients and the wider community, and other agencies in both the statutory and voluntary sector, to achieve improvements in health and more effective service delivery. If successful partnerships are to be established, PCTs will need to ensure that there are effective and early opportunities for organisational and individual learning.

\section{Methods}

The study is designed to assess the organisational and human resource challenges faced by Primary Care Trusts (PCTs) and how they can be met. Specifically the study has four objectives:

1. To specify the organisational and human resources challenges faced by PCTs in fulfilling the roles envisaged in government and local policy;

2. To examine how PCTs are addressing these challenges. In particular, to describe the organisational forms they have adopted, and the OD/HR strategies and initiatives they have planned or in place

3. To assess how effective these structures, strategies and initiatives have been in enabling the PCTs to meet the organisational and human resources challenges they face

4. To identify the factors, both internal to the PCT and in the wider health community, which have contributed to the success or failure of different structures, strategies and initiatives.

It is proposed that the study is undertaken in three main stages:

Stage 1: Preparatory work including a review of key literature in the areas of public sector and NHS organisational development and human resources, and discussions with key researchers and policy makers working in this area.

Stage 2: Detailed case studies in six PCTs designed to examine the organisational and human resources challenges they face.

Stage 3: Analysis of findings from the case studies and identification of key messages for PCTs, Health Authorities and policy makers, and theory development. 


\section{Stage I: Preparatory work}

To provide the context for the study and ensure that the research team takes full account of relevant literature and current research and thinking, the project will begin with a preparatory stage involving both a review of key literature and liaison with other researchers in the field.

\section{Literature review}

The literature relating to both the public and private sector will be examined with particular consideration given to:

- Human resources management and organisational development in the NHS, in particular the research into previous models of primary care commissioning and the development of new NHS organizations;

- The evidence relating to how good HR practice may contribute to the development of effective organizations;

- The literature on models of organisational learning.

The term organisational development (OD) is used rather loosely in the NHS to mean anything from organisational design and restructuring and the implementation of major change programmes to training and individual development activities. An emphasis on the context, as in the notions of receptive and non-receptive contexts for change, [8] leads to consideration of the concepts of organisational learning and the learning organisation, which emphasise internal factors such as leadership, learning culture/climate and the networking and relationships of key groups.[9,10] Application of the organisational learning approach to organisational development in the NHS indicates specific consideration should be given to this area of work.[2] Early work with PCGs suggested that they will wish to adopt more flexible organisational forms and may differ considerably from more traditional NHS organisations.[11] The same study later found an association between the size of PCG and the complexity of management structure. [1] We shall examine the management structures and styles which have emerged in the study PCTs since they were formed in April 2001, with reference to their size, merger history and compexity. It has been suggested that $\mathrm{N}$-form (networking) structures are more likely to support innovation and partnership than M-form (hierarchical) structures.[12,13] Organisational learning theories emphasise lateral communication and dialogue, the ability to draw knowledge and learning from many nodes, and the flexibility to organise in multiple ways to meet requirements. This is seen by some people as replacing a "strategy-structure-system" orientated management philosophy with a more appropriate "purpose-processpeople" one,[14] which, amongst other things, changes the role of the centre from a controlling to an enabling and facilitating one.[15]

\section{Discussions with other researchers and policy maker}

Researchers and policy makers working in the fields of organisational development, human resources management and primary care will have valuable perspectives on the organisational and human resources challenges facing PCTs. Given that PCTs are very new organizations it will be important to consider current thinking as well as published research. In particular, the research team would wish to establish links with the research team undertaking the National Tracker Survey of PCGs and PCTs, and other key researchers in this field.

\section{Defining the challenges}

The information gained from the literature review and discussions with other researchers and policy makers will be used to begin to identify the organisational and human resources challenges that we would expect PCTs to be facing. These are likely to include the need to:

- Develop relationships with colleagues in partner organisations, in particular social services, the local authority, acute trusts and user groups;

- Foster team building and collective responsibility among professionals with limited previous experience of working together, some of whom will remain self-employed;

- Invest in a range of technical and managerial skills in order to fulfill its functions, including service management, health needs assessment, budgeting, strategic planning, data analysis and commissioning, and address issues of management capacity in PCTs;

- Develop robust devolved locality structures within PCTs;

- Ensure that they can recruit and retain an effective workforce;

- Use human resources initiatives to support the modernisation of primary care;

- Deal with the human resources implications of greater joint working between health and social services;

- Manage significant organisational and service change, including Shifting the Balance of Power. [5]

Drawing on this information the research team will draft the interview schedule to be used in the introductory round of interviews with the case study PCTs. The draft 
interview schedule will be piloted with key staff from PCTs who are not part of the case study group.

\section{Stage 2: The case studies}

The core of the study will be based on case studies in six PCTs, designed to observe the process of organisational and human resources development. The criteria that will be used to select the sample PCTs, will be representative of those factors hypothesised to explain differences in organisational structure, including:

- The size of the PCT, which could be measured in terms of population, or number of GPs or GP practices;

- The organisational history of the PCT, particularly whether it has been formed as a result of the merger of two or more PCGs, and at what point this merger had taken place (i.e., prior to Trust status or at the beginning of becoming a Trust);

- The likely complexity of the PCT, measured by the number of directly managed services and the number of PMS practices.

The case studies will focus on three key areas:

- The organisational forms appropriate for PCTs to fulfil their roles - PCTs are new organisations and the evaluation of TPPs and early work with PCGs suggests that they are likely to adopt a number of different organisational forms.[11,16-18] The case studies will be designed to assess how different PCTs decide to organise themselves and to describe how they develop over time. They will also examine how the HR function is provided within or to the PCT.

- The human resources challenges faced by PCTs - PCTs will address these challenges in different ways and with varying levels of priority according to their stage of development and local circumstances. Particular attention will be given to describing the strategies and initiatives they have put in place, assessing how effective these strategies and initiatives have been, and exploring the factors which have contributed to success or failure.

- The areas of organisational learning that PCTs need to address in order to fulfill their functions - Important areas for study here include, the role of the clinician on the executive and evidence of individual, team or group and organisational learning. For example, what is the evidence and what methods or media are in place for, learning within individual GP practices, between practices in the PCT, between professions, between the PCT and other partner agencies in primary healthcare and amongst all organisations concerned in the whole healthcare com- munity. PCTs may be better able to meet their purposes and functions if they are able to demonstrate innovation and learning both from within via development work, exchanging best practice, action learning etc, and from without via benchmarking, stakeholder conferences, joint venturing with other agencies and so on.

The main methods of data collection in the case study PCTs will be semi-structured interviews with key managers and clinicians and group discussions with staff at different levels in the organisations likely to be affected by the OD/HR initiatives adopted by the PCT. Board and Executive members will only be included where they have an $\mathrm{OD} / \mathrm{HR}$ perspective, as relationships between people at the top of NHS trusts have been the subject of a separate study [19] The information from the interviews and group discussions will be supplemented with information gathered from site visits, attendance at key meetings and the examination of local documentation.

Contact with the case study PCTs will be in three phases. Initial contact with the PCTs will focus on testing and refining the ideas developed in stage one of the study, regarding the organisational and human resources challenges facing the PCTs. Information will also be gathered about the structure and organisation of the PCTs, and the research team will begin developing an overview of the human resources initiatives that the PCTs have introduced or plans to introduce in the near future. At this end of this phase, the research team will review the findings from all six PCTs, and select up to three issues in each case study PCT to be explored in greater depth. In the next phase these issues will be followed-up in detail, with particular attention being given to the initiatives or approaches adopted by the PCTs, how these have been planned and implemented, and what they would regard as appropriate measures of success. The final phase of the case studies will be to assess the progress the PCTs have made in implementing the initiatives and where possible assess the impact of they have had on the organisation 6 to 9 months on. The research team will also seek to identify the factors, both internal to the PCT and in the wider health community, which have contributed to the success or failure of different structures, strategies and initiatives.

The six case study PCTs will provide the detailed information for this stage of the study. However, in order to ensure that the findings from the case study PCTs reflect the wider experience of PCG/Ts across the country, a Reference Group of up to 20 other PCG/Ts will be established. The group will also include a number of key officers working in human resources/organisational development and primary care at local, regional, and national level to provide a link to policy forums and a wider 
perspective. Key issues emerging from the case study PCTs will be fed back to this wider group and considered at three workshops to be run at key points during the study. Given the pace of change in primary care at present, the Reference Group will also provide a valuable mechanism for disseminating the interim findings from the research, including models of good practice, to a wider group of PCG/Ts whilst the research is being conducted.

\section{Stage 3: Analysis}

In this final stage of the study, the information gained from Stages 1 and 2 and the feedback from the reference group will be analysed. The analysis will focus on three levels:

- Practical lessons for PCTs - in particular the key organisational and human resources issues which PCTs (particularly newly formed PCTs) need to address; the approaches which have worked well for them; the factors which appear to be important in the success or failure of initiatives; and examples of good HR and OD practice in PCTs.

- Key messages for policy makers - in particular the policy issues which encourage or obstruct good OD and HR practice in PCTs, and the support or guidance which would assist newly formed PCTs or PCGs in their preparatory and early stages.

- Intellectual contributions to the theory of HR and OD, e.g., concerning leadership, motivation and incentives, learning, partnership working and HRM in networks.

To ensure the quality and focus of the research, a local advisory group will be established. The group will meet at key points during the research and will include a PCT Chief Executive, a PCT Chair (or Board Member), and three other members with academic experience in the field of organisational development and primary care. In addition a wider Reference Group will be set up comprising around 20 people who are opinion leaders in primary care OD and HRM and who would help spread the ideas and innovations discovered by the research via formal and informal networks.

During the research the case study PCTs and the wider Reference Group will have a number of opportunities to exchange ideas and good practice. Summaries of emergent themes from each phase of the research will be produced as working papers for dissemination to appropriate audiences. Formative and summative findings will also be disseminated through articles in professional journals and the popular health service press, and opportunities to present findings to relevant conferences and workshops will be pursued.

The foregoing methodological framework is intended to be flexible. The policy context within which the study will be conducted is likely to alter during the course of the study, and qualitative organisational research is itself a dynamic enterprise. Thus, the precise methods and procedures will be chosen in reponse to the preliminary findings of each phase of the research.

\section{Competing interests}

None declared.

\section{Acknowledgements}

Thanks to Judith Smith and Rod Sheaff for comments on the draft protocol.

\section{References}

I. Regen E, Smith J, Goodwin N, McLeod H, Shapiro J: Passing on the baton: final report of a national evaluation of primary care groups and trusts. Birmingham: Health Services Management Centre; 200I

2. Attwood $M$ : Organisation development in health care: reflections on a decade of practice. In: Managing Medicine : A Survival Guide Edited by Brown J, Sanderson D. pp. 285-296. London: Financial Times Healthcare; 1997285-296

3. NHS Executive: Primary care Groups: Taking the Next Steps. Working Together: Human Resources guidance and requirements for Primary Care Trusts. London; 1999

4. Street A, Place M: The management challenges for Primary Care Groups. London: Kings Fund; 1998

5. DoH: Shifting the Balance of Power within the NHS. London: Department of Health; 200 I

6. DoH: The New NHS: modern, dependable. London: Department of Health; 1997

7. Mays N, Goodwin N, Malbon G, Leese B, Mahon A, Wyke S: What were the achievements of total purchasing pilots in their first year and how can they be explained? London: King's Fund Publishing; 1998

8. Pettigrew A, Ferlie E, McKee L: Shaping Strategic Change. London: Sage; 1992

9. Senge P: The Fifth Discipline: The Art and Practice of Learning Organisations. New York: Doubleday Currency; 1991

10. Pedler MJ, Burgoyne JG, Boydell TH: The Learning Company: A strategy for sustainable development., Second Edition edn. Maidenhead: McGrawHill; 1997

II. Smith J, Regen E, Goodwin N, McLeod H, Shapiro J: Getting into their stride: interim report of a national evaluation of primary care groups. Birmingham: Health Services Management Centre; 2000

12. Hedlund G: Assumptions of Hierarchy and Hetrarchy: An Application to the Multinational Corporation. In: Organisational Theory and the Multinational Corporation Edited by Ghoshall S, Westney E. London: Macmillan; 1993

13. Hedlund G: A Model of Knowledge Management and the $\mathbf{N}$ form Corporation. Strategic Management Journal 1994, 1 5:73-90

14. Bartlett C, Ghoshall S: Beyond Strategy, Structure, Systems to Purpose, Process, People. In: The Relevance of a Decade Edited by Duffy P. Boston: Harvard Business School; 1994

15. Ferlie E, Pettigrew A: The Nature and Transformation of Corporate Headquarters. Journal of Management Studies 1996, 33:495523

16. Mays N, Goodwin N, Bevan G, Wyke S: Total Purchasing: a profile of the national pilot projects. London: Kings Fund; 1997

17. Place M, Newbronner E: The Role, Functions and Costs of PCTs: a case study from East Yorkshire. York: York Health Economics Consortium; 1999

18. Wilkin D, Gilliam S, Leese B: The National Tracker Survey of Primary Care groups and Trusts. Progress and Challenges 1999/2000. Manchester: National Primary Care Research and Development Centre; 2000

19. Robinson R, Exworthy M: Two at the top: a study of working relationships between chairs and chief executives at health authorities, boards and trusts in the NHS. London: IHSM; 1999 